\title{
Evolution of binaries with compact objects in globular clusters
}

\author{
Natalia Ivanova \\ Dept. of Physics, University of Alberta, 11322-89 Ave, Edmonton, AB, T6G 2E7, Canada \\ email: nata.ivanova@ualberta.ca
}

\begin{abstract}
Dynamical interactions that take place between objects in dense stellar systems lead to frequent formation of exotic stellar objects, unusual binaries, and systems of higher multiplicity. They are most important for the formation of binaries with neutron stars and black holes, which are usually observationally revealed in mass-transferring binaries. Here we review the current understanding of compact object's retention, of the metallicity dependence on the formation of low-mass X-ray binaries with neutron stars, and how mass-transferring binaries with a black hole and a white dwarf can be formed. We discuss as well one old unsolved puzzle and two new puzzles posed by recent observations: what descendants do ultra-compact X-ray binaries produce, how are very compact triples formed, and how can black hole low-mass X-ray binaries acquire non-degenerate companions?
\end{abstract}

Keywords. binaries : close, globular clusters: general

\section{Introduction}

A low-mass X-ray binary (LMXB) is a binary star consisting of a neutron star (NS) or a black hole $(\mathrm{BH})$ accretor, with a less-massive donor that fills its Roche lobe (RL). A donor can be a main-sequence (MS) star, a white dwarf (WD) or a red giant (RG). An isolated binary (a field binary) can become an LMXB via several possible scenarios (e.g., Bhattacharya \& van den Heuvel 1991; Verbunt 1993; Tauris \& van den Heuvel 2006). A typical formation scenario starts with a common envelope event, where the initially most massive star overfills its RL. The expulsion of the formed common envelope leads to the binary orbit tightening. Later, the initially most massive star explodes in a core-collapse (CC) Ib or Ic supernova and the compact object is formed. Further orbit's shrinkage can take place due to tides, magnetic braking and gravitational waves in, likely, an eccentric binary; a second episode of a common envelope event can take place as well. When the initially less massive stars overfills its RL, the system is revealed as an X-ray source. It is essential that a typical formation scenario of a field LMXB includes several phases or processes which are not yet fully understood - tides, common envelope events, magnetic braking and natal supernova kicks (see, e.g., reviews Eggleton 2006; Tauris \& van den Heuvel 2006; Zahn 2008; Knigge et al. 2011; Ivanova et al. 2013). We further distinguish LMXBs by how they appear - whether they have a stable disk accretion and therefore can be seen persistently, or if they raise drastically their X-ray luminosity in outbursts, and therefore are transient sources.

Globular clusters (GCs) contain about two orders of magnitude more LMXBs per stellar mass than the Milky Way (MW) population contains of "native" LMXBs (Grindlay 1984; Verbunt \& van den Heuvel 1995; Bildsten \& Deloye 2004). This overabundance has been attributed to the role that dynamical encounters play in the formation of LMXBs in GCs. Indeed, studies have shown strong positive correlation between the stellar encounter rate and the number of close X-ray binaries in GCs (Pooley et al. 2003; Bahramian et al. 
2013). Additionally, the metallicity dependence was found - that a metal-rich GC in the Galaxy and in M31 is $~ 3$ times more likely to contain a bright LMXB than a metal-poor GC (Grindlay 1993; Bellazzini et al. 1995). Observations of GCs around distant galaxies have also shown that the fraction of GCs hosting LMXBs is larger by a factor of 3 in metal-rich than in metal-poor GCs (Kundu et al. 2002; Sarazin et al. 2003; Kim et al. 2006; Sivakoff et al. 2007; Paolillo et al. 2011; Kim et al. 2013).

It is interesting that most of the very uncertain physics involved in the formation of "native" field LMXBs - e.g., common envelope phase, binary modification due to natal supernova kicks, tides - is either not involved in the formation scenario of a dynamically formed LMXB, or affects the formation scenario with a different, often lesser, impact. For example, supernova kicks affect GCs LMXBs mainly by reducing the retention of compact objects in GCs after their formation (Verbunt \& Hut 1987; Drukier 1996). The formation path of an LMXB in a GC, on the other hand, can take a short-cut by pairing a NS or a $\mathrm{BH}$ via a dynamical encounter. To understand the puzzles that observations bring us, we need to analyze how the dynamical formation channels - binary exchanges, tidal captures, physical collisions and triple formations - perform with different type of donors.

\section{Formation and retention of compact objects}

The mean three-dimensional pulsar birth velocity is $400 \mathrm{~km} / \mathrm{s}$, and a pulsar twodimensional speed can be as large as $1600 \mathrm{~km} / \mathrm{s}$ (Hobbs et al. 2005). The escape velocity from a GC is an order of magnitude less than pulsars' mean kick velocities. If all NSs are born with kicks we derived for pulsars, only a tiny fraction of them could remain in a GC (Pfahl et al. 2002). Indeed, a typical GC with current mass of $2 \times 10^{5} M_{\odot}$ and Kroupa's initial mass function (Kroupa 2002), forms about 3000 NSs. If the escape velocity is $40 \mathrm{~km} / \mathrm{s}$, only one NS can be retained if all stars were single at birth (Ivanova et al. 2008b). Being born in a binary changes the post-kick system's space velocity (e.g., Brandt \& Podsiadlowski 1995; Pfahl et al. 2002), and if all stars would be initially in binaries, a $2 \times 10^{5} M_{\odot}$ GC could retain $15 \mathrm{NSs}$ (Ivanova et al. 2008b). This is still too little to explain the observations. Indeed, Ter 5 , which is about $2 \times 10^{6} M_{\odot}$, is estimated to contain 150 recycled pulsars (Bagchi et al. 2011).

The problem is considered now to be resolved by assuming that the observed high pulsar velocities are only related to the case of a CC supernova. Indeed, there is another formation channel for NSs, electron capture (EC) supernova, that takes places when a degenerate $\mathrm{ONeMg}$ core reaches $1.38 M_{\odot}$ (Miyaji et al. 1980; Nomoto 1984; Timmes \& Woosley 1992). EC supernova takes place in a narrow range of masses in single stars, where this range depends slightly on metallicity and the used stellar code (for example, this range is $7.7-8.3 M_{\odot}$ at $Z=0.02$ and $6.2-6.8 M_{\odot}$ at $\mathrm{Z}=0.0005$ using the STARS/ev code, Pols et al. 1995). In binary stars, the range of initial stellar masses that can produce EC supernova is larger, due to mass transfer history (Podsiadlowski et al. 2004). In addition, for stars in binaries, EC NSs can be formed due to accretion on a companion WDs, or due to a merger of two WDs. As a result, in field, 10-15\% of all NSs could be formed via various EC channels (Ivanova et al. 2008b). Probably the most famous example is Crab Supernova (Kitaura et al. 2006; Tominaga et al. 2013). The most important feature of EC supernovae for GCs is that the explosion is much weaker (e.g., Dessart et al. 2006), and hence their kicks are believed to be weaker as well. Under assumption of lower kicks, most of retained NSs in a GC come from different EC supernovae channels. The ratio of CC NSs to EC NSs in a GC is about 1 to $30-200$ instead of about 10 to 1 
in the field (Ivanova et al. 2008b). Since post-EC NS masses are low, $1.22-1.27 M_{\odot}$, a low-mass dominated NSs mass function can be expected in GCs.

The understanding of BHs retention has recently drastically changed. It can be esti-

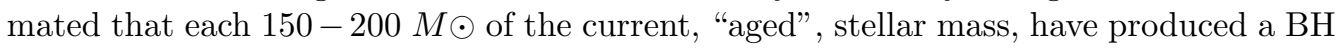
in the past. This is assuming Kroupa's initial mass function and no mass loss from a GC except that due to stellar evolution. Due to lower kicks, the retention fraction immediately after formation is much higher than for NSs, 30-40\%, if the escape velocity from a GC is $50 \mathrm{~km} / \mathrm{s}$ (Belczynski et al. 2006). It was considered previously that equipartition between the stars in a GC would lead to the formation of a $\mathrm{BH}$ sub-cluster in a center of a GC (Spitzer instability), followed by rapid BH population depletion via an ejection of nearly all BHs (Spitzer 1969; Kulkarni et al. 1993). However, recent detailed numerical simulations of $\mathrm{BH}$ subclusters have shown that up to $20 \%$ of the $\mathrm{BHs}$ may remain in massive BH subclusters, and these subclusters do not reach equipartition (O'Leary et al. 2006). Monte Carlo simulations of a whole GC have shown that up to $25 \%$ of initial BHs can remain and participate in interactions with other stars (Downing et al. 2010), or that even more than a half of formed BHs can remain, with no evidence for the Spitzer instability (Morscher et al. 2013). It can be now assumed that a large number of initially retained BHs can remain in GCs, 10-60\%, depending on cluster mass and its initial virial radius (Morscher et al. 2014).

\section{Formation channels and metallicity dependence}

The observed metallicity dependence for bright LMXBs can be interpreted as a combination of the metallicity dependence due to the encounter rates, and the metallicity dependence due to the mass transfer - whether the formed LMXBs appear as persistent or transient sources, how bright they are when 'on', and how long they can remain as bright sources.

Typical dynamical formation channels of a binary with a compact object are: (i) via binary exchanges (BEs), (ii) physical collisions (PCs) with a giant or (iii) via tidal captures (TCs) (e.g., see Verbunt \& Lewin 2006, and references therein). A dynamical event provides a direct LMXB formation if a newly formed binary can start the mass transfer within the Hubble time while evolved in isolation. Not all formed binaries with a NS and a BH will become LMXBs directly, but the formed binaries can serve as seed binaries for further dynamical encounters (hardening, triples formation) and eventually can also start the mass transfer.

For MW GCs, bright LMXBs are those that have their X-ray luminosity $L_{X}>$ $10^{36} \mathrm{erg} \mathrm{s}^{-1} .18$ bright transient or persistent LMXBs have been observed in MW GCs. For the 13 known by 2004, a compilation is provided in Verbunt \& Lewin 2006. Two more transients were found in Ter 5 (Bordas et al. 2010; Bahramian et al. 2014), one more in NGC 6440 (Heinke et al. 2010), and the first transient was found in each of two GCs, NGC 6388 and M 28 (Bozzo et al. 2011; Eckert et al. 2013; Papitto et al. 2013).

The separation between metal-rich (also known as red) and metal-poor (also known as blue) MW GCs is not strictly defined, and is usually taken at $[\mathrm{Fe} / \mathrm{H}]=-1$ (a gap in the distribution of metallicities is observed between $[\mathrm{Fe} / \mathrm{H}]=-1.2$ and $[\mathrm{Fe} / \mathrm{H}]=-0.8$, and this gap disappears if the sample is limited to low-reddening clusters, Vanderbeke et al. 2014a,b). Here, we will refer to the GCs that have $[\mathrm{Fe} / \mathrm{H}]$ between -1.5 and -0.5 as "intermediate" metallicity GCs, if $[\mathrm{Fe} / \mathrm{H}]<-1.5$ as "distinctly metal-poor" GCs, and if $[\mathrm{Fe} / \mathrm{H}]>-0.5$ as "distinctly metal-rich" GCs.

From 18 known bright MW GCs LMXBs only 2 are in "distinctly metal-poor" GCs (both are in M 15), 8 are in the "intermediate" metallicity GCs (LMXBs in M 28, 
NGC 1851, NGC 6388, NGC 6652, NGC 6712, Ter 1, Ter 2, Ter 6) and another 8 are in "distinctly metal-rich" GCs (LMXBs in Ter 5, NGC 6440, NGC 6441, NGC 6624, Lil 1), where two GCs contain more than one bright LMXB (Ter 5 and NGC 6440).

If we look at "classes" of bright MW LMXBs by their donor-type (note that this is also strongly linked to their orbital periods), we can see that 6 likely have a WD donor (Verbunt \& Lewin 2006; Zurek et al. 2009; Altamirano et al. 2010). These binaries are called ultra-compact X-ray binaries (UCXBs). 3 of these UCXBs are in "intermediate" GCs (NGC 1851, NGC 6712, Ter 2), one in a "distinctly metal-poor" GC (M 15) and two in "distinctly metal-rich" GCs (NGC 6624, NGC 6440). 2 of bright MW LMXBs likely have MS donors, both are persistent and are located in GCs with $[\mathrm{Fe} / \mathrm{H}]>-1$ (NGC 6441, Bałucińska-Church et al. 2004, NGC 6652, Engel et al. 2012). 5 of bright LMXBs have most likely either a subgiant or a giant companion (Verbunt \& Lewin 2006; Testa et al. 2012; Papitto et al. 2013), one in a "distinctly metal-poor" GC (M 15), two in "distinctly metal-rich" GCs (Ter 5, NGC 6440) and two are in the "intermediate" GCs (M 28, Ter 6). In addition, 5 bright LMXBs are transients where neither the companion nor orbital period has yet been determined, all of which are located in metal-rich GCs with $[\mathrm{Fe} / \mathrm{H}] \gtrsim-1$. Among LMXBs where we have some knowledge of the donor, most are persistent ( 8 persistent versus 5 transient LMXBs). Transient sources tend to have a longer orbital period, both for non-degenerate donors (4 out of 5 LMXBs with giant donors are transient, while all LMXBs with MS donors are persistent) and degenerate donors (the UCXB with the largest orbital period, in NGC 6440, is transient).

As we can see, for MW GCs, the 3 times greater probability of hosting an LMXB by a red GC than by a blue GC is cumulative. The ratio would change depending on what is defined as a metal-poor and a metal-rich GC, and is not satisfied for any class of donors separately. The most striking difference is for LMXBs with MS donors, but this is based on two LMXBs only.

Studies of extragalactic GCs have shown a similar preference for LMXBs to reside in metal-rich GCs. The ratio was found to hold across the range of X-ray luminosities, from $2 \times 10^{37} \mathrm{erg} \mathrm{s}^{-1}$ to $5 \times 10^{38} \mathrm{erg} \mathrm{s}^{-1}$ (Kim et al. 2013). For even higher X-ray luminosity, where the accretors are likely BHs, this ratio is less certain, but still is above one, $2.5_{-1.1}^{+0.9}$. There, it also was shown that the metallicity effect is not affected by other factors such as stellar age, GC mass, stellar encounter rate, and galacto-centric distance. The caveat in making a link between MW GCs LMXBs and extragalactic ones is that the the boundary between blue and red GCs is not the same, and for extragalactic GCs is done using colors (e.g., Brodie \& Strader 2006), not [Fe/H] as for MW GCs. The relation between colors and $[\mathrm{Fe} / \mathrm{H}]$ is however non-linear (Vanderbeke et al. 2014b).

It is important also that the population of detected extragalactic LMXBs is not similar to the population of bright GC LMXBs in our Milky Way. Indeed, in the Galaxy, the brightest observed GC UCXB 4U1820-303, in NGC 6624, has $L_{x} \approx 4-7 \times 10^{37} \mathrm{erg} \mathrm{s}^{-1}$ (Giacconi et al. 1974; van der Klis et al. 1993) (we note that this LMXBs could be special in other respects too, see $\S 4$ ). In extragalactic GC LMXBs, statistically significant studies were done for LMXBs above $L_{x} \approx 2 \times 10^{37} \mathrm{erg} \mathrm{s}^{-1}$ (Kim et al. 2013). Different ranges of X-ray luminosity are dominated by different donors (e.g., Fragos et al. 2008). For example, mass transfer in binaries with MS donors is driven by magnetic braking, and rarely produce X-ray luminosity $\gtrsim 2 \times 10^{37} \mathrm{erg} \mathrm{s}^{-1}$ (Fragos et al. 2008, 2009). Hence, MS donors most likely are not present among the X-ray sources that form X-ray luminosity functions of extragalactic GCs.

Among LMXBs, the evolution of UCXBs is best understood; predominantly they will be seen as bright persistent sources (e.g., Deloye \& Bildsten 2003; Bildsten \& Deloye 2004), although there are some discrepancies between theoretically expected and 
observationally derived mass-transfer rates for UCXBs with longer orbital periods (van Haaften et al. 2012b; Cartwright et al. 2013; Heinke et al. 2013).

The strongest uncertainty for extragalactic GC LMXBs is coming from our limited knowledge of the duty cycles in LMXBs with giant donors, and their luminosity during outburst (see overview of the problem in Fragos et al. 2008). Further, their outburst duration can be longer than the history of X-ray astronomy, and hence observationally they would be known as persistent. There are several X-ray binaries in the galaxy which confirm this suspicion as their outburst duration is at least 20 years - for example, GRS 1915+105 which turned on 1992 and has been active ever since (Castro-Tirado et al. 1992; Deegan et al. 2009), or the X-ray binary in Ter 1, which was active for about 20 years and then turned off between 1996 and 1999 (Guainazzi et al. 1999).

LMXBs with RG donors are most likely to be formed via BE encounters; the evolutionary expansion of a giant brings the system to the start of a mass transfer. The BEs formation channel does not have a metallicity dependence for the encounter rates (for GCs which otherwise are "dynamical twins"). The mass transfer slightly favors the appearance of metal-rich LMXBs - those can have the short duration of a persistent mass transfer, while metal-poor LMXBs with RG donors are likely always in quiescence (Ivanova et al. 2012). It is interesting that the observations of MW GCs show the opposite situation to theoretical expectations: the only persistent RG-LMXB is located in a metal-poor cluster M 15, although this can be related to the uncertainty in our understanding of the duty cycles.

For LMXBs with MS donors, the formation mechanisms include BEs and TCs. TCs, unlike BEs, can provide direct LMXB formation in most of the formed binaries, though cumulatively are less efficient for LMXBs formation than BEs (Ivanova et al. 2008b). In close NS-MS binaries, the most effective mechanism of orbital shrinkage is the magnetic braking, which operates only in stars with an outer convective zone. TCs are also more effective with stars that are convective (Portegies Zwart \& Meinen 1993). However, mostmassive MS stars in metal-poor GCs lack surface convective zone (Ivanova 2006). This slight difference between stars which are convective at the surface, and those which are radiative, for a small mass-range, affects the following: (i) the rate of the orbital shrinkage, where MS-NS binaries with a larger post-BE or post-TC orbital period can start the MT if their MS star is metal-rich; (ii) the formation rate via TCs, which are more efficient in metal-rich GCs; and (iii) metal-poor MS-LMXBs are expected to be in quiescence, where persistent MS-LMXBs are expected to be observed only in metal-rich globular clusters. The dynamically formed NS-MS binaries that would have started the mass transfer if the donor is metal-rich and have failed to start the mass-transfer with a metal-poor donor, will start the MT when the donor leaves the MS.

The best-understood formation mechanism of GC LMXBs is for UCXBs - via PC of a NS with a RG (as was initially proposed in Verbunt 1987, and see most recent details in Ivanova et al. 2005; Lombardi et al. 2006). If PC is between a NS and a subgiant, it often leads to a direct LMXB formation (Ivanova et al. 2005). In UCXBs, post-PC binary orbital evolution is solely due to angular momentum loss via gravitational wave radiation, same mechanism operates during the mass transfer, and there is no known effects through which the metallicity could affect the appearance of the X-ray binary during the mass transfer. The theoretically predicted number of UCXBs is consistent with the observed bright LMXBs in MW GCs, in case if at least several per cent of ever formed NSs is retained Ivanova et al. (2005).

In extragalactic GC LMXBs, which are generally more luminous than "bright" LMXBs in MW GCs, the potential donors are likely RGs and WDs. For neither of the donors, as discussed above, metallicity affects the formation rate per giant. The dynamical properties 
of blue and red extragalactic GCs in the studied sample were found to be statistically identical (Kim et al. 2013). The total formation rate depends however also on the number of encounter participants, which are NSs and RGs. Theoretically, it is expected that slightly more of NSs can be formed in a metal-poor GC than in a similar metal-rich GC (Ivanova et al. $2008 \mathrm{~b}$ ) - this trend is opposite to the observed metallicity dependence.

From stellar evolution, we know that metal-poor MS stars evolve faster than metalrich MS stars of the same mass. Therefore, metal-poor GCs of the same age have stars of a lower mass at their MS turn-off. Lifetime of the RG stage is shorter for metal-poor stars as well. Since RGs live shorter, a metal-poor GCs should contain RGs that have a smaller mass range than RGs in a metal-rich GCs. If one assumes that GCs of different metallicities had the same initial mass function, detailed calculations confirm that the fraction of RG stars that a metal-poor GC can have is about twice less than a metal-rich GC has (Ivanova et al. 2012). This directly affects the encounter rates, and increases it in a metal-rich GC, compared to a metal-poor GC, by a factor of two.

The mass of the RG also plays a role: first, the expulsion of a RG envelope, which is more massive in a metal-rich RG, results in a smaller post-PC separation, and in a direct LMXB formation in a larger fraction of encounters; and second, the effective cross-section - how likely each RG is to participate in a dynamical encounter - is higher in metal-rich GCs. In total, this results in about 3 times more frequent formation of UCXBs in metalrich clusters than in metal-poor, and can explain the observed metallicity dependence (Ivanova et al. 2012). The RG lifetime affects RG-LMXBS as well, as the duration of a RG-NS mass transfer is shorter in metal-poor GCs.

Between all GC LMXB classes, the best theoretical understanding, as well as the best agreement on numbers with the observations of MW GC LMXBs, we have is for the formation and evolution of UCXBs. It is thought that millisecond pulsars (MSPs) are related to LMXBs, and transitional systems between LMXBs and binary MSPs (bMSPs) are now known (e.g., Wijnands \& van der Klis 1998; Archibald et al. 2009; Papitto et al. 2013). Simulations of GCs have shown good agreement between the number and period distribution for all theoretically produced radio bMSPs, but with the striking exception for radio bMSPs presumably produced by UCXBs (Ivanova et al. 2008a). The lifetime of an UCXB at $L_{x}>10^{36} \mathrm{erg} \mathrm{s}^{-1}$ is $\tau \sim 10^{8} \mathrm{yr}$. This implies that for each currently observed bright UCXB, 100 time more bMSPs should have been created, or about 600 bMSPs with a small orbital period and a low-mass companion should have been detected in MW GCs. However, we do not observe such a radio bMSP population in GCs, nor in the overall number of MSPs, nor for expected companion-mass and orbital period parameter space for bMPSs. This problem is by no means confined only to GCs, there is a lack of post-UCXBs radio bMSPs in the galactic "field" population as well (Deloye 2008).

The interesting case that can provide in future a clue on the fate of post-UCXB systems is the discovery of PSR J1719-1438 - a "field" MSP which has the orbital period of 2.2 hour, its companion mass is about that of Jupiter, but the minimum companion density is $23 \mathrm{~g} \mathrm{~cm}^{-3}$, suggesting that it may be an ultralow-mass carbon white dwarf (Bailes et al. 2011). This system is very puzzling, as it takes more than a Hubble time to get to this period under conventional UCXB evolution (van Haaften et al. 2012a), while this evolution can be deemed possible if additional donor wind, irradiation and donor's evaporation are taken into account (Benvenuto et al. 2012). While this may explain why we do not see post-UCXB system as bMSPs, it still does not explain the mismatch in the total number of MSPs. On the other hand, it is plausible that NSs that were spun up in an UCXB binary are less likely to be detected in radio, and instead can be detected 
in $\gamma$-rays, as, for example, in case of PSR J1311-3430 that has a $93 \mathrm{~min}$ orbital period and very low-mass helium companion (Pletsch et al. 2012; Romani et al. 2012).

\section{Role of triples}

In a hierarchical triple, a distant third body exerts tidal forces on the inner binary. As a result, there is a cyclic exchange of the angular momentum between an inner binary and a third body, causing variations in the eccentricity and inclination of the stars orbits (Kozai 1962; Ford et al. 2000; Blaes et al. 2002). If Kozai mechanism is coupled with dissipative tidal friction (Kozai Cycle with Tidal Friction, KZTF), the inner binary can be driven to start the RL overflow of one of the companions, with the subsequent either a merger or a start of the stable mass transfer (Eggleton \& Kisseleva-Eggleton 2006; Fabrycky \& Tremaine 2007). If the timescale of KZTF is shorter than the timescale of dynamical encounters, KZTF can provide a formation channel for LMXBs in GCs.

The interesting case is LMXB 4U 1820-303 in NGC 6624. Binary orbital period of this system is $\sim 685 \mathrm{~s}$ (Stella et al. 1987; Anderson et al. 1997). Theoretically predicted period increase for this system, if it is a binary, is $\dot{P} / P>8 \times 10^{-8} \mathrm{yr}^{-1}$ (Rappaport et al. 1987). However, from observations, the orbital period is decreasing as $(\dot{P} / P)_{\text {obs }}=$ $-(5.3 \pm 0.3) \times 10^{-8} \mathrm{yr}^{-1}$ (van der Klis et al. 1993; Peuten et al. 2014). In addition, 4U 1820303 has the luminosity variation by a factor of $\sim 2$ at a super-orbital period $P \sim 170 \mathrm{~d}$ (Chou \& Grindlay 2001). Chou \& Grindlay (2001) have suggested that this system could be a hierarchical triple with a 1.1 day outer orbital period.

Prodan \& Murray (2012) have found that they can explain this system to be a triple, where the inner binary is composed of $1.4 M_{\odot} \mathrm{NS}$ and $0.067 M_{\odot} \mathrm{WD}$, and the third companion has mass of $0.55 M_{\odot}$. However, the outer semi-major axis is small, $1.5 R_{\odot}$, and the outer orbital period is only $0.15 \mathrm{~d}$. The re-analysis of simulations performed in Ivanova et al. (2008b) have shown that none of the dynamically formed triples with a NS was anywhere close to the observed compactness, with the tightest dynamically formed triple having its outer orbital period about a day, and most of dynamically formed triples were even much wider. This should come as no surprise, as an inner binary in a dynamically formed hierarchical triple would be rarely substantially tighter than the tightest of the two binaries that have participated in the encounter. Too tight binaries both have a small encounter rate and can be easily destroyed via collisions during an encounter (Fregeau et al. 2004). A possible explanation is that this hierarchical triple, formed initially dynamically, was substantially wider than now, and then was tightened through either external common envelope event, initiated by an outer companion, or a double common envelope event. The latter is a bit less likely, also in that case the seed inner WD-NS binary could not have been formed via a PC.

Prodan \& Murray (2014) had also suggested that 3 more GC UCXBs could be triple systems. This, if confirmed, would bring the fraction of triple UCXBs to $2 / 3$ of all MW GC UCXBs. It also may suggest that yet unknown formation channel is as efficient as the current favorite formation channel of UCXBs - PC, or that most of NS-WD binary, formed initially via a PC, form subsequently a triple system. How plausible is that? Theoretically expected formation rates suggests that each NS binary has a $5 \%$ chance to form a hierarchically stable triple in a dense GC like 47 Tuc per Gyr, and as much as $15 \%$ chance per Gyr in a cluster like Ter 5 . So, in a dense cluster, most of seed NS binaries could become a member of a triple system at some point (Ivanova 2008, also reanalysis of simulations presented in Ivanova et al. 2008b). The formation of the triples with very short outer orbital periods is however as difficult dynamically as for the described above 4U 1820-303. 


\section{LMXBs with BH accretors}

Observational frequency of very bright extragalactic GC LMXBs, with $L_{x} \gtrsim 10^{39}$ erg $\mathrm{s}^{-1}$ indicating possible $\mathrm{BH}$ accretors, is about $0.7 \div 2 \times 10^{-9}$ per $M_{\odot}$ in massive GCs with an average $M_{\mathrm{V}} \approx-9$ (Kim et al. 2006; Sivakoff et al. 2007; Humphrey \& Buote 2008; Kim et al. 2013). BH LMXBs formed via binary exchanges are likely to have low duty cycles and not be detected as bright LMXBs (Kalogera et al. 2004; Fragos et al. 2008, 2009). TCs by a BH likely destroy the capturing star (Kalogera et al. 2004). This leaves as most likely donors WDs. A BH-WD LMXB spends an order of $5 \times 10^{5}$ yr at $L_{x} \gtrsim 10^{39}$ erg $\mathrm{s}^{-1}$. Assuming that about $10 \%$ of all formed BHs is retained, the observationally inferred formation rate of LMXBs is $\sim 4 \times 10^{-3}$ per Gyr per each retained BH.

It has been shown that PCs with giants, the most effective mechanism to form NSWD LMXBs, can not provide direct formation of BH-WD LMXBs at the observationally inferred rate. A dynamically formed binary with a $\mathrm{BH}$ is several times more massive than any non-BH binary in a GC. If a dynamically formed BH-WD binary participates in a binary-binary encounter, the chance to form a hierarchically stable triple is substantial. If Kozai timescale is short compared to the characteristic time this triple has before its next dynamical encounter, triple induced mass transfer can transform the inner BH-WD binary into LMXBs (Ivanova et al. 2010). Interestingly, Maccarone et al. (2010) have found a large X-ray flux variation in RZ 2109, such that this source is consistent with being in a triple. RZ 2109 was the first discovered extragalactic GC X-ray source which is believed to contain a stellar mass BH (Zepf et al. 2008).

The observations of bright BH LMXBs in extragalactic GCs can be extrapolated to our MW GCs. It is important that after the "bright" stage at $L_{x} \gtrsim 10^{39} \mathrm{erg} \mathrm{s}^{-1}$, an ultra-compact X-ray BH-WD binary will remain a mass-transferring system for many gigayears. With uniform formation rate, and average GC age of $10 \mathrm{Gyr}$, an average BH-WD LMXB will exist $\sim 10^{4}$ times longer than a bright BH-WD LMXB, and will spend most of its life at extremely low mass transfer rates. The expected frequency per dense and massive MW GCs is then of order $10^{-5}$ per $M_{\odot}$. This implies that almost every massive GC in the Galaxy might contain a very faint BH-WD LMXB! Note that only $7 \mathrm{MW}$ GCs are above $M_{\mathrm{V}}=-9$, to have $\mathrm{BHs}$ retention comparable to massive extragalactic GCs.

The recent radio observations of MW GCs clusters have indeed indicated that some of them contain faint potential BH-LMXBs. Five candidates are under investigation two LMXBs in M22 (Strader et al. 2012), one in M62 (Chomiuk et al. 2013), in 47 Tuc (Miller-Jones et al. in prep.) and in one more GC (Shishkovsky et al. in prep). Interesting that only in two cases WD companions are plausible, in M22 and in 47 Tuc. The rest of the companions are likely non-degenerate - MS stars, or giants, or "red stragglers". A BH LMXB with a non-degenerate donor can continue mass transfer at a very low rate for several gigayears, comparable to that of $\mathrm{BH}-\mathrm{WD}$ binaries, hinting that the dynamical formation rate of $\mathrm{BH}$ binaries with non-degenerate donors is comparable to that of BH UCXBs. The formation booster with help of triples has not yet been shown to work effectively for non-degenerate companions. Therefore, the puzzle of the formation of BH LMXBs with non-degenerate companions in GCs remains to be explained.

\section{References}

Altamirano D. et al., 2010, ApJL, 712, L58

Anderson S. F. et al., 1997, ApJL, 482, L69

Archibald A. M. et al., 2009, Science, 324, 1411

Bagchi M., Lorimer D. R., \& Chennamangalam J., 2011, MNRAS, 418, 477

Bahramian A. et al., 2014, ApJ, 780, 127 
Bahramian A., Heinke C. O., Sivakoff G. R., \& Gladstone J. C., 2013, ApJ, 766, 136

Bailes M. et al., 2011, Science, 333, 1717

Bałucińska-Church M., Church M. J., \& Smale A. P., 2004, MNRAS, 347, 334

Belczynski K., Sadowski A., Rasio F. A., \& Bulik T., 2006, ApJ, 650, 303

Bellazzini M. et al., 1995, ApJ, 439, 687

Benvenuto O. G., De Vito M. A., \& Horvath J. E., 2012, ApJL, 753, L33

Bhattacharya D. \& van den Heuvel E. P. J., 1991, Phys.Rep., 203, 1

Bildsten L. \& Deloye C. J., 2004, ApJL, 607, L119

Blaes O., Lee M. H., \& Socrates A., 2002, ApJ, 578, 775

Bordas P. et al., 2010, The Astronomer's Telegram, 2919, 1

Bozzo E. et al., 2011, A\& $A, 535, \mathrm{~L} 1$

Brandt N. \& Podsiadlowski P., 1995, MNRAS, 274, 461

Brodie J. P. \& Strader J., 2006, ARAA, 44, 193

Cartwright T. F. et al., 2013, ApJ, 768, 183

Castro-Tirado A. J., Brandt S., \& Lund N., 1992, IAU circular, 5590, 2

Chomiuk L. et al., 2013, ApJ, 777, 69

Chou Y. \& Grindlay J. E., 2001, ApJ, 563, 934

Deegan P., Combet C., \& Wynn G. A., 2009, MNRAS, 400, 1337

Deloye C. J., 2008, in AIP Conference Series, Vol. 983, 40 Years of Pulsars, Bassa C., Wang Z., Cumming A., Kaspi V. M., eds., pp. 501-509

Deloye C. J. \& Bildsten L., 2003, ApJ, 598, 1217

Dessart L. et al., 2006, ApJ, 644, 1063

Downing J. M. B., Benacquista M. J., Giersz M., \& Spurzem R., 2010, MNRAS, 407, 1946

Drukier G. A., 1996, MNRAS, 280, 498

Eckert D. et al., 2013, The Astronomer's Telegram, 4925, 1

Eggleton P., 2006, Evolutionary Processes in Binary and Multiple Stars

Eggleton P. P. \& Kisseleva-Eggleton L., 2006, Ap\&SS, 304, 75

Engel M. C. et al., 2012, ApJ, 747, 119

Fabrycky D. \& Tremaine S., 2007, ApJ, 669, 1298

Ford E. B., Kozinsky B., \& Rasio F. A., 2000, ApJ, 535, 385

Fragos T. et al., 2008, ApJ, 683, 346

Fragos T. et al., 2009, ApJL, 702, L143

Fregeau J. M., Cheung P., Portegies Zwart S. F., \& Rasio F. A., 2004, MNRAS, 352, 1

Giacconi R. et al., 1974, ApJ Supp, 27, 37

Grindlay J. E., 1984, Advances in Space Research, 3, 19

Grindlay J. E., 1993, in ASP Conference Series, Vol. 48, The Globular Cluster-Galaxy Connection, Smith G. H., Brodie J. P., eds., p. 156

Guainazzi M., Parmar A. N., \& Oosterbroek T., 1999, A\&A, 349, 819

Heinke C. O. et al., 2010, ApJ, 714, 894

Heinke C. O. et al., 2013, ApJ, 768, 184

Hobbs G., Lorimer D. R., Lyne A. G., \& Kramer M., 2005, MNRAS, 360, 974

Humphrey P. J. \& Buote D. A., 2008, ApJ, 689, 983

Ivanova N., 2006, $A p J, 636,979$

Ivanova N., 2008, in Multiple Stars Across the H-R Diagram, Hubrig S., Petr-Gotzens M., Tokovinin A., eds., p. 101

Ivanova N. et al., 2010, ApJ, 717, 948

Ivanova N. et al., 2012, ApJL, 760, L24

Ivanova N., Heinke C. O., \& Rasio F. A., 2008a, in AIP Conference Series, Vol. 983, 40 Years of Pulsars, Bassa C., Wang Z., Cumming A., Kaspi V. M., eds., pp. 442-447

Ivanova N. et al., 2008b, MNRAS, 386, 553

Ivanova N. et al., 2013, Astronomy and Astrophysics Review, 21, 59

Ivanova N. et al., 2005, ApJL, 621, L109

Kalogera V., King A. R., \& Rasio F. A., 2004, ApJL, 601, L171

Kim D.-W. et al., 2013, ApJ, 764, 98 
Kim E. et al., 2006, ApJ, 647, 276

Kitaura F. S., Janka H.-T., \& Hillebrandt W., 2006, A\&AA, 450, 345

Knigge C., Baraffe I., \& Patterson J., 2011, ApJ Supp, 194, 28

Kozai Y., 1962, AJ, 67, 591

Kroupa P., 2002, Science, 295, 82

Kulkarni S. R., Hut P., \& McMillan S., 1993, Nature, 364, 421

Kundu A., Maccarone T. J., \& Zepf S. E., 2002, ApJL, 574, L5

Lombardi, Jr. J. C. et al., 2006, ApJ, 640, 441

Maccarone T. J., Kundu A., Zepf S. E., \& Rhode K. L., 2010, MNRAS, 409, L84

Miyaji S., Nomoto K., Yokoi K., \& Sugimoto D., 1980, PASJ, 32, 303

Morscher M. et al., 2014, ArXiv e-prints

Morscher M., Umbreit S., Farr W. M., \& Rasio F. A., 2013, ApJL, 763, L15

Nomoto K., 1984, ApJ, 277, 791

O'Leary R. M. et al., 2006, ApJ, 637, 937

Paolillo M. et al., 2011, ApJ, 736, 90

Papitto A. et al., 2013, Nature, 501, 517

Peuten M., Brockamp M., Küpper A. H. W., \& Kroupa P., 2014, ApJ, 795, 116

Pfahl E., Rappaport S., \& Podsiadlowski P., 2002, ApJ, 573, 283

Pletsch H. J. et al., 2012, Science, 338, 1314

Podsiadlowski P. et al., 2004, ApJ, 612, 1044

Pols O. R., Tout C. A., Eggleton P. P., \& Han Z., 1995, MNRAS, 274, 964

Pooley D. et al., 2003, ApJL, 591, L131

Portegies Zwart S. F., \& Meinen A. T., 1993, A\&BA, 280, 174

Prodan S. \& Murray N., 2012, ApJ, 747, 4

Prodan S. \& Murray N., 2014, ArXiv e-prints

Rappaport S., Ma C. P., Joss P. C., \& Nelson L. A., 1987, ApJ, 322, 842

Romani R. W. et al., 2012, ApJL, 760, L36

Sarazin C. L. et al., 2003, ApJ, 595, 743

Sivakoff G. R. et al., 2007, ApJ, 660, 1246

Spitzer, Jr. L., 1969, ApJL, 158, L139

Stella L., Priedhorsky W., \& White N. E., 1987, ApJL, 312, L17

Strader J. et al., 2012, Nature, 490, 71

Tauris T. M. \& van den Heuvel E. P. J., 2006, Formation and evolution of compact stellar X-ray sources

Testa V. et al., 2012, A\&GA, 547, A28

Timmes F. X. \& Woosley S. E., 1992, ApJ, 396, 649

Tominaga N., Blinnikov S. I., \& Nomoto K., 2013, ApJL, 771, L12

van der Klis M. et al., 1993, A\&A, 279, L21

van Haaften L. M., Nelemans G., Voss R., \& Jonker P. G., 2012a, A\&̛A, 541, A22

van Haaften L. M., Voss R., \& Nelemans G., 2012b, A\&SA, 543, A121

Vanderbeke J. et al., 2014a, MNRAS, 437, 1725

Vanderbeke J. et al., 2014b, MNRAS, 437, 1734

Verbunt F., 1987, ApJL, 312, L23

Verbunt F., 1993, ARAA, 31, 93

Verbunt F. \& Hut P., 1987, in IAU Symposium, Vol. 125, The Origin and Evolution of Neutron Stars, Helfand D. J., Huang J.-H., eds., p. 187

Verbunt F. \& Lewin W. H. G., 2006, Globular cluster X-ray sources, Lewin W. H. G., van der Klis M., eds., pp. 341-379

Verbunt F. \& van den Heuvel E. P. J., 1995, X-ray Binaries, 457

Wijnands R. \& van der Klis M., 1998, Nature, 394, 344

Zahn J.-P., 2008, in EAS Publications Series, Vol. 29, EAS Publications Series, Goupil M.-J., Zahn J.-P., eds., pp. 67-90

Zepf S. E. et al., 2008, ApJL, 683, L139

Zurek D. R. et al., 2009, ApJ, 699, 1113 